\title{
A Study on Relapsed $\beta$-Cell Lymphoma in Elderly Patient of Bangladeshi Population with Rituximab, Gemcitabin and Oxaliplatin: an Effective Salvage Regimen
}

\author{
*ME Hoque ${ }^{1}$, S Karim² ${ }^{2}$, RS Giasuddin ${ }^{3}$, MMR Siddiqui ${ }^{3}$, M Setu ${ }^{4}$ \\ ${ }^{1}$ Md Ehteshamul Hoque, Department of Oncology, Anwer Khan Modern \\ Medical College Hospital, Dhaka, Bangladesh \\ ${ }^{2}$ Dr. Shahnaz Karim, Department Of Transfusion Medicine \\ Dhaka Medical College Hospital, Dhaka, Bangladesh. \\ ${ }^{3}$ Dr. Rubayet Shekh Giasuddin \& Dr. Md. Mahmudur Rahman Siddiqui, Department of \\ Medicine, Anwer Khan Modern Medical College Hospital, Dhaka, Bangladesh \\ ${ }^{4}$ Dr. Muntahina Setu, Department of Paediatrics, Anwer Khan Modern Medical \\ College Hospital, Dhaka, Bangladesh
}

*Corresponding author

\begin{abstract}
High-dose therapy (HDT) with stem-cell support is the reference treatment for relapsed lymphoma, but is not appropriate for all patients. In Bangladesh High-dose therapy with stem cell support is not yet available. Conventional salvage chemotherapies have been used with limited efficacy and significant toxicity. Rituximab, gemcitabin and oxaliplatin are active as single agents in relapsed or refractory lymphoma, and have demonstrated synergistic effects in vitro and in vivo. Twenty two patients with relapsed or refractory or $\beta$-cell lymphoma received up to Six cycle of R-GemOx (rituximab $375 \mathrm{mg} / \mathrm{m}^{2}$ on day 1 , gemcitabine $1000 \mathrm{mg} / \mathrm{m}^{2}$ and oxaliplatin $100 \mathrm{mg} / \mathrm{m}^{2}$ on day 2 ). The majority $(60 \%)$ had diffuse large $\beta$-cell lymphoma. After four cycle of $\mathrm{R}-\mathrm{GemOx}$, the overall response rate was $90 \%$ (45\% complete response $(\mathrm{CR}) /$ unconfirmed $\mathrm{CR}(\mathrm{CRu})$. High $\mathrm{CR} / \mathrm{CRu}$ rates were observed in all histological subtypes. In patients who had previously received rituximab, the CR/CRu rate after six cycles was $70 \%$. The 2 year event-free and overall survival rates (median follow-up of 24 months) were 55\%, respectively, among responders, the probability of being disease free for 2 years was $70 \%$. Treatment was generally well tolerated. R-GemOx shows promising activity with acceptable toxicity in patients with relapsed/refractory $\beta$-cell lymphoma.
\end{abstract}

Key words: gemcitabine, lymphoma, oxaliplatin, R-GemOx, rituximab

\section{Introduction}

Multi agent chemotherapy has been a major advanced in the treatment of non-Hodgkin's lymphoma. Although some patients can be cured with this approach, disease relapse and refractory disease constitute significant problems for the treatment of all histological subtypes of lymphoma. To date, high-dose therapy (HDT) with hematological stem-cell support is the reference treatment for patients with chemo sensitive relapsed aggressive lymphoma ${ }^{1}$. In relapsed indolent lymphoma, this approach also seems superior to conventional salvage chemotherapy ${ }^{2}$. However, many patients cannot benefit from HDT as a result of advanced age, significant co-morbidities previous use of HDT or resistance to salvage chemotherapy. Conventional salvage regiments without HDT, such as dexamethasone, cytarabine and cisplatin (DHAP) or etoposide, methylprednisolone, cytarabine and cisplatin, are associated with poor long-term disease control and significant toxicity ${ }^{3}$. Thus, regiments based on innovative 
drug combinations with better efficacy and less toxicity are needed for the management of patients with advanced lymphoma who are not eligible for HDT. Gemcitabine offers advantages over its parent compound, cytarabine, in terms of delivery of highly effective intracellular concentrations ${ }^{4}$. Gemcitabine has demonstrated single-agent efficacy in relapsed or refractory aggressive lymphoma, including mantle cell lymphoma $(\mathrm{MCL})^{5-7}$. The anti-CD20 monoclonal antibody, rituximab, has also shown single-agent activity in lymphoma and can enhance the efficacy of standard chemotherapy regimen in the first and second line setting ${ }^{8-11}$. In addition, the platinum derivative oxaliplatin has similar efficacy to cisplatin, with improved renal safety ${ }^{12,13}$ and reduced induction of chemo resistance ${ }^{14}$. The favorable safety profile of oxaliplatin makes this agent potentially suitable for elderly patient with co-morbidities. The mechanistic synergy and non-overlapping toxicity profiles of rituximab, gemcitabine and oxaliplatin (R-GemOx) indicate that combination regimens containing these three agents may offer advantages over conventional regimens in terms of efficacy, safety and tolerability. Based on these considerations, we conducted study of the combination of RGemOx in the treatment of patients with relapsed or refractory $\beta$-cell lymphoma. As in Bangladesh context, where HDT with stem cell support is not available.

\section{Materials and methods}

\section{Patient's selection and evaluation}

Patients were treated at the Oncology Department of Anwer Khan Modern Medical College \& Hospital, Dhaka, United Hospital Ltd, Dhaka and City Hospital Ltd, Dhaka. Patients with recurrent or refractory CD20 positive lymphoma of any performance status were eligible for inclusion if there were serious co morbidities, relapsed \& refractory after having conventional R-CHOP or CHOP therapy. Patient was evaluated before treatment by clinical examinations, computed tomography (CT) scans of the thorax, abdomen and pelvis, and bone marrow trephine biopsy. All patients underwent blood sampling for complete blood counts and routine chemistry, including assessment of hepatic and renal function and measurement of lactate dehydrogenase levels.

\section{Treatment protocol}

Rituximab $375 \mathrm{mg} / \mathrm{m}^{2}$ was admitted on day 1 according to the standard infusion rate escalation protocol16. Premeditation with methylprednisolone $6 \mathrm{mg} / \mathrm{kg}$ i.v, acetaminophen $1000 \mathrm{mg}$ orally and dexchlopheniramine $6 \mathrm{mg}$ orally was administered to avoid infusion related side-effects. Gemcitabine $1000 \mathrm{mg} / \mathrm{m}^{2}$ (in $500 \mathrm{ml}$ of normal saline) was administered on day 2 , at a fixed dose rate of $10 \mathrm{mg} / \mathrm{m}^{2} / \mathrm{min}$. This prolonged administered schedule has shown to achieve superior intracellular drug concentrations than the standard $30 \mathrm{~min}$ i.v. schedule $^{17}$. Oxaliplatin $100 \mathrm{mg} / \mathrm{m} 2$ over $2 \mathrm{~h}$ was administered on day 2 after gemcitabine. Cycles were repeated every 14 days. A complete blood count was carried out on days 7, 10 and 14 of each treatment cycle to assess hematological toxicity. Patients underwent clinical examination and routine chemistry assessment before each new cycle. No dose adjustment was planned in the event of hematological toxicity, but cycles were delayed until the absolute neutrophil count reached $1.0 \times 10 / 1$ and the platelet count reached $100 \times 10 / 1$. No dose adjustment of oxaliplatin was required in the event of peripheral neuropathy.In the event of abnormal results by neurological examination or if a patient experienced significant paresthesia lasting for 14 days or more, oxaliplatin was to be stopped until symptoms improved and then restarted at a same dose of $100 \mathrm{mg} / \mathrm{m}^{2}$. In the event of pharyngolaryngeal dysesthesia, the duration of the oxaliplatin infusion was to be prolonged from 2 to $6 \mathrm{~h}$. Primary prophylaxis with Granulocyte Colony-Stimulating Factor (GCSF) was administered with subsequent cycle to aid maintenance of the dose intensity.

\section{Toxicity and response assessments}

Hematological and non-hematological toxicity evaluation was conducted on day 1 of each treatment cycle and included neurological examination and laboratory assessment with 
complete blood cell count and serum chemistry tests. Thoracic, abdominal and pelvic CT scans and bone marrow biopsy (in patients with bone marrow involvement at initial diagnosis) were conducted to assess response after both induction therapy and completion of consolidation therapy. After four treatment cycle (induction therapy), patient achieving a complete response (CR), unconfirmed complete response $(\mathrm{CRu})$ or partial response $(\mathrm{PR})$ were eligible for to Two further cycles of R-GemOx (consolidation therapy).

\section{Statistical methods}

The primary end point of the study was the overall response rate (ORR) after four cycles of treatment. Secondary end points were event-free survival (EFS), defined as the time interval from the date of enrollment in the study until disease progression, relapse or death whichever occurred first and overall survival (OS) calculated from the date of enrolment until death from any cause. For patients who responded after four cycles, time to relapse was defined as the time interval from the date of evaluation until the date of progression or relapse whichever came first. Survival curves were estimated using the product-limit method of Kaplan-Meier and compared using the log-rank test. Relative dose intensity (RDI) for gemcitabine and oxaliplatin was calculated according to Hryniuk et al.18. The Fisher's exact test was used for comparisons. Statistical tests were considered significant when the twosided $\mathrm{P}$ value was $<0.05$. Confidence intervals (CIs) were computed with a $95 \%$ coverage; CI binominal exact bounds were computed for proportions.

\section{Results}

From January 2008 to July 2011, 22 patients were enrolled and were eligible for analysis. Median follow-up was 24 months (data cut-off November 30, 2011). Pretreatment patient characteristics are summarized in Figure 1.

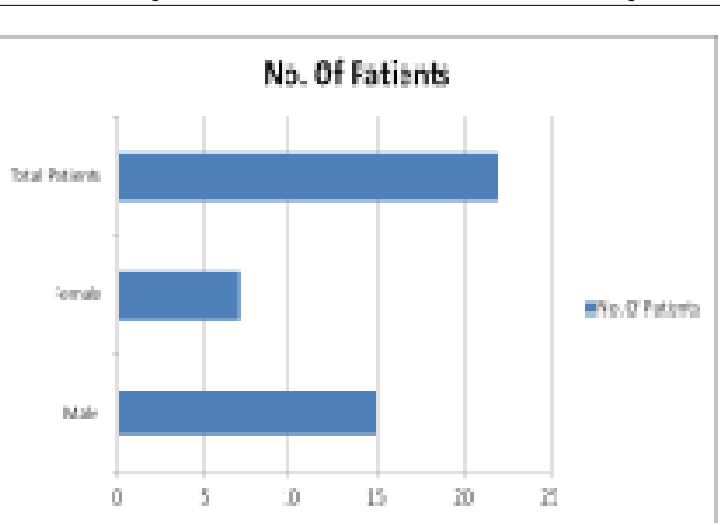

Figure 1: Number of patients in the study

\section{Treatment exposure}

The overall number of cycles administered was 132 (range, 1-6). Most patients received the intended number of cycles and the median duration of exposure was 12 weeks. No dose reductions were required for rituximab or gemcitabine or oxaliplatin.Seven patients (32\%) required a dose delay, most commonly because of peripheral neuropathy and gastrointestinal upset. 3 patients required G-CSF. Of the 110 cycles considered for this analysis (first cycles were excluded), 88 (80\%) were delivered on time and at the planned dose. The average RDI was $84 \%$ for gemcitabine and $82 \%$ for oxaliplatin. Treatment was delayed in a total of 22 cycles; 17 cycles (16\%) were delayed because of peripheral neuropathy or neutropenia or thrombocytopenia or gastrointestinal upset and 5 cycles (4\%) were delayed because of nonhematological toxicity (febrile neutropenia in four cases and cardiac failure in one case). In total, 20 patients $(91 \%)$ completed the planned six cycles. Reasons for stopping treatment were severe cardiac failure (one patient) and patient's request (one after five cycles).

\section{Response to treatment}

Response rates were calculated after induction therapy (four cycles) and at completion of therapy for patients who completed six cycles. 2 patients progressed during the induction phase. After four cycles, 10 patients achieved a CR, 6 had a CRu and 4 had a PR, resulting in an ORR $(\mathrm{CR}+\mathrm{CRu}+\mathrm{PR})$ of $90 \%(95 \% \mathrm{CI}, 75 \%$ to 95\%). Response rates were similar for the following three histological subtypes: diffuse large B-cell lymphoma (DLBCL), 82\% (95\% 
CI, 65\% to 93\%); follicular lymphoma (FL), $75 \%$ (95\% CI, $35 \%$ to $97 \%$ ) and MCL, $100 \%$ (95\% CI, to $100 \%)$. A trend towards a better response rate to induction therapy was observed for rituximab-naive patients compared with those previously treated with rituximab with (95\% versus $73 \%$, respectively; $\mathrm{P}=0.11$ ). Response rates were lower among patients who had experienced no response to their last treatment or response duration $<1$ year than among patients who had previous response duration $>1$ year $(53 \%$ versus $97 \%$, respectively; $\mathrm{P}<0.001)$. At the end of treatment, 15 patients $(70 \%$; $95 \% \mathrm{CI}, 57 \%$ to $84 \%$ ) had achieved a $\mathrm{CR} / \mathrm{CRu}$, one patient $(4 \%)$ had a PR and 6 patients progressed, translating into an ORR of $90 \%$.

\section{Event-free and overall survival}

At the time of this analysis (medium duration of follow-up 24 months for the 20 patients who responded to induction treatment), No patients had relapsed, translating into a 2-year progression-free survival of $70 \%(95 \% \mathrm{CI}$, $44 \%$ to $85 \%$ ). For the responders with DLBCL, No relapsed. Of note, for the 19 responders not previously treated with rituximab, the probability of remaining relapse free at 2 years was $81 \%(95 \% \mathrm{CI}, 61 \%$ to $100 \%)$ compared with $37 \%$ (95\% CI, $7 \%$ to $68 \%$; P < 0.05 ) for the 19 patients previously treated with rituximab. Kaplan-Meier curves for OS and EFS are shown in Figure 1. With a median follow-up of months, the 2 years EFS and OS rates were $43 \%$ (95\% CI, $27 \%$ to $60 \%$ ) and $66 \%$ (95\% CI, $50 \%$ to $82 \%$ ), respectively. The median time to progression (TtP) was 22 months (range, 1-24 months). No patient died during the treatment period. Among patients with DLBCL, the 2 years EFS was $42 \%$ (95\% CI, $22 \%$ to $62 \%$ ) and the median TtP was 24 months, with no significant difference between patients previously treated with rituximab and rituximabnaive patients (median TtP of 16 and 24 months, respectively).

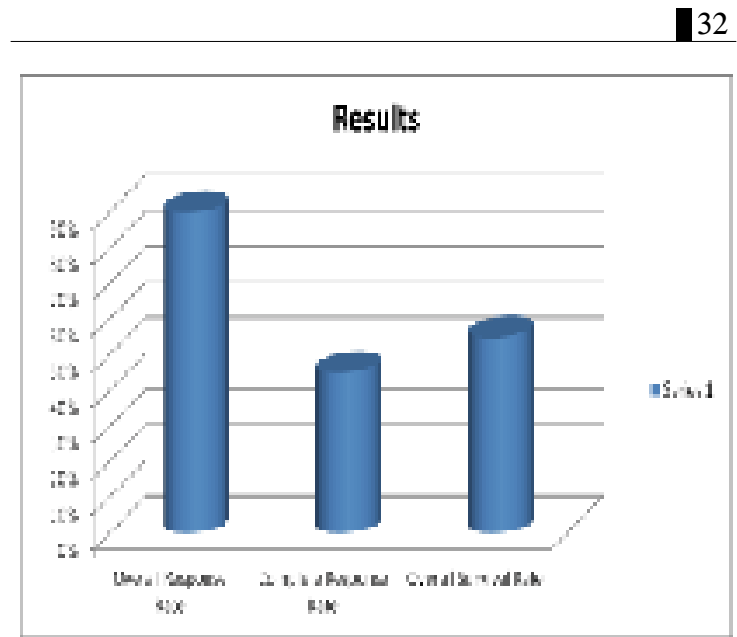

Figure 2: Outcome of the Study on relapsed B-cell lymphoma in elderly patient of Bangladeshi population with Rituximab, Gemcitabin and Oxaliplatin.

\section{Safety}

No fatal toxicity was observed. Treatment was generally well tolerated, with the majority of patients hospitalized for only one night with the first administration of rituximab during the first cycle of treatment. Neutropenia grades 2, 3 and 4 were reported in $33 \%, 22 \%$ and $11 \%$ of cycles, respectively, while thrombocytopenia grades 2,3 and 4 were reported in $12 \%, 19 \%$ and $4 \%$ of cycles, respectively. Febrile neutropenia was observed in $4 \%$ of cycles. Grade 2 neutrotoxicity occured in $45 \%$ of cycles, but no grade $3 / 4$ neutrotoxiicty was reported. Two patients $(4 \%)$ received red cell transfusions with a median of 1 (range, 2-6) .No renal toxicity was observed. No other grade $3 / 4$ non-hematological toxicity was observed.

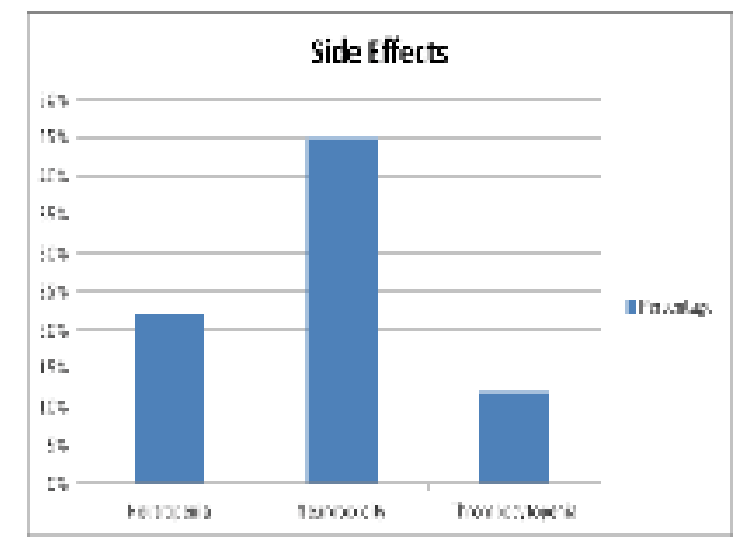

Figure 3: Side effects during study. 


\section{Discussion}

In this study of 22 patients with relapsed or refractory $\beta$-cell lymphoma, four cycles of $\mathrm{R}$ GemOx achieved a high ORR of $90 \%$. These results compare favorable with data for conventional chemotherapy regimens, which show low response rates and few durable responses ${ }^{3}$. Our results also similer with those of other combinations of rituximab and chemotherapy in the ralapsed/ refractory setting: Kewalramani et al. 10 reported a 90\% ORR and $70 \%$ CR rate in a population of 20 younger patients treated with rituximab, ifosamide, carboplatin and etoposide, none of whom had been previously exposed to rituximab. Jermann et al,11 reported a $68 \%$ ORR and $28 \%$ CR rate with the rituximab, etopocide, doxorubicin, vincristine, cyclophosphamide and prednisolone regimen in a population of 50 patients among which $4 \%$ had receive prior rituximab. At the end of treatment, very high $\mathrm{ORR}$ and $\mathrm{CR} / \mathrm{CRu}$ rates were seen in patients who had not previously received HDT (90\% and $70 \%$, respectively). The R-GemOx regimen had a very favorable toxicity profile. No nephrotoxicity was seen, and hematological toxicity was manageable with the help of growth factor support. Rates of compliance and delivery of the intended number of cycle at the intended dose were good. There were few infections and no deaths. Oxaliplatin-associated neurotoxicity occurred in only $45 \%$ of cycles and no grade $3 / 4$ neurotoxicity was observed.These results are particularly encouraging for the treatment of elderly patients with lymphoma. Each component of the R-GemOx regimen may contribute to its efficacy; indeed, the results of this study support a synergistic or supraadditive action for rituximab when combined with gemcitabine and oxaliplatin. This observation is consistent with results from previous student in lymphoma and other cancers. The use of R-GemOx is currently under further investigation in a larger, multicenter study being conducted by the group d'Etudes Lymphomes de I'Adulte in patients with relapsed/refractory $\beta$-cell lymphoma, with DLBCL. In conclusion, the $\mathrm{R}-\mathrm{GemOx}$ regimen shows promising activity with an acceptable toxicity profile, and may be a favorable treatment option for patients of Bangladesh with relapsed/refractory $\beta$ cell lymphoma as High-dose therapy with stem cell support is not yet available.

\section{Conclusion}

In this study of 22 patients with relapsed or refractory B-cell lymphoma, four cycles of RGemOx achieved a high ORR of $90 \%$. In Bangladesh we know that stem cell support with HDT is not available. So, for elderly with comorbid, relapsed cases of $\beta$-cell lymphoma is difficult to manage. This study is encouraging for such population of patient in Bangladesh. This result also support favorable data for conventional chemotherapy regimen for which show low response rate and few durable responses $^{3}$. The $\mathrm{R}-\mathrm{GemOx}$ regimens have a very favorable toxicity profile. Haematotoxicity was well managed with G-CSF. Neurotoxicity was negligible. There was no serious infection. No treatment related death was found. R-gemox therapy for elderly relapsed and refractory $\beta$-cell lymphoma is encouraging with good response and acceptable toxicity profile. Furthur study may be encouraged to treat $\beta$-cell lymphoma patient with R-GemOx therapy in other settings including initial treatment also.

\section{References}

1. Philip T, Guglielmi C, Hagenbeek A, et al. Autologous bone marrow transplantation as compared with salvage chemotherapy in relapses of chemotherapy-sensitive non-Hodgkin's lymphoma. N Engl J Med 1995; 333:1540-1545.

2. Schouten HC, Qian W, Kvaloy S et al. High-dose therapy improves progression free survival and survival in relapsed follicular non-Hodgkin's lymphoma: results from the randomized European CUP trial. J Clin Oncol 2003; 21: 3918-3927.

3. Rodriguez-Monge EJ, Cabanillas F. Long-term follow-up of platinum-based lymphoma salvage regimens. The M.D. Anderson Cancer Center experience. Hematol Oncol Clin North Am 1997; 11: 937-947.

4. Storniolo AM, Allerheiligen SR, Pearce HL. Preclinical, pharmacologic, and phase I studies of gemcitabine. Semin Oncol 1997; 24: S7-2-S7-7.

5. Dumontet C, Morschhauser F, Solal-Celigny $\mathrm{P}$ et al. Gemcitabine as a single agent in the treatment of relapsed or refractory low-grade non-Hodgkin's lymphoma. Br J Haematol 2001; 113: 772-778. 
6. Fossa A, Santoro A, Hiddemann $W$ et al. Gemcitabine as a single agent in the treatment of relapsed or refractory aggressive non-Hodgkin's lymphoma. J Clin Oncol 1999; 17: 3786-3792.

7. Savage DG, Rule SA, Tighe M et al. Gemcitabine for relapsed or resistant lymphoma. Ann Oncol 2000; 11: 595-597.

8. Marcus $\mathrm{R}$, Imrie $\mathrm{K}$, Belch $\mathrm{A}$ et al. CVP chemotherapy plus rituximab compared with CVP as first-line treatment for advanced follicular lymphoma. Blood 2005; 105: 1417-1423.

9. Coiffier B, Lepage E, Briere $\mathrm{J}$ et al. CHOP chemotherapy plus rituximab compared with $\mathrm{CHOP}$ alone in elderly patients with diffuse large-B-cell lymphoma. N Engl J Med 2002; 346: 235-242.

10. Kewalramani $\mathrm{T}$, Zelenetz $\mathrm{AD}$, Nimer $\mathrm{SD}$ et al. Rituximab and ICE as second-line therapy before autologous stem cell transplantation for relapsed or primary refractory diffuse large B-cell lymphoma. Blood 2004; 103: 3684-3688.

11. Jermann M, Jost LM, Taverna $C$ et al. Rituximab$\mathrm{EPOCH}$, an effective salvage therapy for relapsed, refractory or transformed B-cell lymphomas: results of a phase II study. Ann Oncol 2004; 15: 511-516.

12. Chau I, Webb A, Cunningham D et al. An oxaliplatinbased chemotherapy in patients with relapsed or refractory intermediate and high-grade non-Hodgkin's lymphoma. Br J Haematol 2001; 115: 786-792.
13. Machover D, Delmas-Marsalet B, Misra SC et al. Dexamethasone, high-dose cytarabine, and oxaliplatin (DHAOx) as salvage treatment for patients with initially refractory or relapsed non-Hodgkin's lymphoma. Ann Oncol 2001; 12:1439-1443.

14. Raymond E, Chaney SG, Taamma A, et al. Oxaliplatin: a review of preclinical and clinical studies. Ann Oncol 1998; 9: 1053-1071.

15. Jaffe ES, Harris NL, Stein H, et al. Pathology and genetics of tumours of haematopoietic and lymphoid tissues. In (IARC) IAfRoC (ed): World Health Organization Classification of Tumours. Lyon, France: IARC Press 2001.

16. Coiffier B, Haioun $\mathrm{C}$, Ketterer $\mathrm{N}$ et al. Rituximab (anti-CD20 monoclonal antibody) for the treatment of patients with relapsing or refractory aggressive lymphoma: a multicenter phase II study. Blood 1998; 92: 1927-1932.

17. Grunewald R, Kantarjian H, Keating MJ et al. Pharmacologically directed design of the dose rate and schedule of 2\#,2\#-difluorodeoxycytidine (gemcitabine) administration in leukemia. Cancer Res 1990; 50: 6823-6826.

18. Hryniuk W, Ragaz J, Peters W. Dose density by any other name. J Clin Oncol 2004; 22: 750-751; author reply 751-753. 